\title{
Renoprotective Effects of a Selective Estrogen Receptor Modulator, Raloxifene, in an Animal Model of Diabetic Nephropathy
}

\author{
Alexis Dixon ${ }^{\mathrm{a}}$ Corinne C. Wells ${ }^{\mathrm{a}}$ Sandhya Singh ${ }^{\mathrm{a}}$ Regina Babayan ${ }^{\mathrm{a}}$ \\ Christine Maric ${ }^{a, b}$ \\ a Department of Medicine and ${ }^{\mathrm{b} C e n t e r}$ for the Study of Sex Differences in Health, Aging and Disease, \\ Georgetown University Medical Center, Washington, D.C., USA
}

\section{Key Words}

Diabetes, kidney $\cdot$ Raloxifene $\cdot$ Glomerulosclerosis $\cdot$

Tubulointerstitial fibrosis

\begin{abstract}
Background/Aims: Our previous studies have shown that supplementation with $17 \beta$-estradiol $\left(E_{2}\right)$ from the onset of diabetes attenuates diabetic nephropathy. However, $E_{2}$ is accompanied by feminizing effects as well as adverse side effects on other organs. The current study examined the renoprotective effects of a selective estrogen receptor modulator, raloxifene (RAL), in an experimental model of diabetic nephropathy. RAL activates estrogen receptors and estrogenreceptor-mediated cellular events without the side effects of $E_{2}$. Methods: The study was performed in Sprague-Dawley nondiabetic (ND), streptozotocin-induced diabetic (D) and streptozotocin-induced D + RAL rats ( $n=6 /$ group). Results: After 12 weeks of treatment, $D$ was associated with increased urine albumin excretion (ND: $4.2 \pm 0.4 ; \mathrm{D}: 41.3 \pm 9.0 \mathrm{mg} /$ day), glomerulosclerosis [glomerulosclerotic index; ND: 0.26 $\pm 0.04 ; \mathrm{D}: 1.86 \pm 0.80$ arbitrary units (AU)], tubulointerstitial fibrosis (tubulointerstitial fibrosis index; ND: $0.37 \pm 0.05$; D: $2.12 \pm 0.50 \mathrm{AU}$ ), increased collagen type I [ND: $1.31 \pm 0.07$; $\mathrm{D}: 4.65 \pm 0.09$ relative optical density (ROD)], collagen type IV (ND: $0.64 \pm 0.03 ; \mathrm{D}: 1.37 \pm 0.11 \mathrm{ROD})$ and transforming growth factor beta (TGF- $\beta$ ) protein expression (ND: $0.65 \pm$ 0.08 ; D: $1.25 \pm 0.10 \mathrm{ROD}$ ), increased density of CD68-posi-
\end{abstract}

tive cells (ND: $1.37 \pm 3.02$; D: $29.2 \pm 1.74$ cells $/ \mathrm{mm}^{2}$ ) and increased plasma levels of interleukin-6 (ND: $14.8 \pm 5.0$; D: $51.3 \pm 14.0 \mathrm{pg} / \mathrm{ml}$ ). Treatment with RAL partially or fully attenuated these processes (urine albumin excretion: $21.0 \pm$ $5.0 \mathrm{mg} /$ day; glomerulosclerotic index: $0.40 \pm 0.06 \mathrm{AU}$; tubulointerstitial fibrosis index: $0.20 \pm 0.04 \mathrm{AU}$; collagen type I: $2.55 \pm 0.49$ ROD; collagen type IV: $0.70 \pm 0.09$ ROD; TGF- $\beta$ : $0.91 \pm 0.08$ ROD; CD68: $6.03 \pm 2.38$ cells $/ \mathrm{mm}^{2}$; interleukin6: $31.2 \pm 5.0 \mathrm{pg} / \mathrm{ml}$ ). Conclusions: Our data indicate that treatment with RAL attenuates albuminuria and renal structural changes associated with diabetes.

Copyright $\odot 2007$ S. Karger AG, Basel

\section{Introduction}

Diabetes is associated with severe end-organ complications including diabetic nephropathy, one of the leading causes of end-stage renal failure [1-3]. Epidemiological data suggest that women with diabetes are at a 5-fold greater risk of developing cardiovascular disease compared to nondiabetic women [4-6]. While the data regarding the risk of development and progression of renal disease in diabetic compared with nondiabetic women are not as definitive, mainly because it has not been adequately analyzed thus far, it appears that in the setting of diabetes, women exhibit a greater risk of developing renal disease compared with nondiabetic women $[4,5,7$,

\section{KARGER}

Fax +4161306 1234 E-Mail karger@karger.ch www.karger.com
(C) 2007 S. Karger AG, Basel

0250-8095/07/0272-0120\$23.50/0

Accessible online at:

www.karger.com/ajn
Christine Maric, $\mathrm{PhD}$

Center for the Study of Sex Differences in Health, Aging and Disease

Georgetown University Medical Center, 394 Building D, 4000 Reservoir Road NW

Washington, DC 20057 (USA)

Tel. +1 202687 5363, Fax +1 202687 4210, E-Mail cm255@georgetown.edu 
8]. These observations suggest that in the setting of diabetes, the mechanisms that protect women from developing renal disease are no longer effective. It is generally thought that female sex hormone, namely estrogen, affords protection against the development of cardiovascular and renal disease $[4,5,7,8]$. Our recent findings show that diabetes in females is associated with reduced levels of circulating estradiol [9], suggesting that the loss of the female gender as a protective factor against the development of cardiovascular and renal disease in diabetes may be due to the imbalance of female sex hormones and their receptor expression in target tissues [9].

These observations led us to perform studies on the effects of $17 \beta$-estradiol $\left(E_{2}\right)$ in an animal model of diabetic nephropathy that have shown that supplementation with $\mathrm{E}_{2}$ from the onset of diabetes attenuates albuminuria, glomerulosclerosis and tubulointerstitial fibrosis associated with diabetic nephropathy [10]. This renoprotective effect of $E_{2}$ appears to be a direct effect on cell proliferation and extracellular matrix (ECM), the two major cellular processes adversely regulated in diabetic nephropathy. However, regardless of the fact that $\mathrm{E}_{2}$ supplementation appears to offer renoprotection in diabetic nephropathy, $\mathrm{E}_{2}$ has been reported to increase the risk of developing breast cancer [11], and if administered in males, may result in unwanted feminizing side effects. Thus, the most effective way of affording $\mathrm{E}_{2}$-mediated renoprotection would be via eliminating its feminizing and organ side effects.

Raloxifene (RAL), a selective estrogen receptor modulator (SERM), activates estrogen receptors and induces estrogen-receptor-mediated cellular events without the side effects of $E_{2}$ [12]. RAL has been shown to reduce mesangial cell proliferation and collagen synthesis in vitro [13], while in vivo, RAL has been shown to ameliorate renal damage in a model of type 2 diabetes [14]. However, the mechanisms by which RAL exerts renoprotection in diabetes are largely unknown. Thus, the aim of the present study was to examine the renoprotective effects of RAL in the streptozotocin-induced model of diabetic nephropathy.

\section{Materials and Methods}

\section{Animals}

The study was performed in female Sprague-Dawley rats (Harlan, Madison, Wisc., USA; 10 weeks of age). All animals were maintained on a phytoestrogen-free rat chow (Harlan) and allowed free access to tap water. The animals were randomly divided into 3 treatment groups: nondiabetic (ND, $n=6)$, diabetic
$(\mathrm{D}, \mathrm{n}=6)$, and diabetic treated with raloxifene $(\mathrm{D}+\mathrm{RAL}, \mathrm{n}=6)$. RAL was administered from the onset of diabetes at a dose of 10 $\mathrm{mg} / \mathrm{kg} /$ day in the phytoestrogen-free chow. These animals received RAL-containing chow to achieve a dose of $10 \mathrm{mg} / \mathrm{kg} /$ day and were then given phytoestrogen-free chow ad libitum.

Diabetes was induced by a single intraperitoneal injection of $55 \mathrm{mg} / \mathrm{kg}$ streptozotocin (Sigma, St. Louis, Miss., USA) in $0.1 \mathrm{M}$ citrate buffer ( $\mathrm{pH} 4.5)$ after an overnight fast. ND animals were injected with $0.1 \mathrm{M}$ citrate buffer only. All D animals were given daily subcutaneous injections of insulin (2-4 U, Lantus, Aventis Pharmaceuticals Inc., Kansas City, Miss., USA) to maintain blood glucose levels between 250 and $400 \mathrm{mg} / \mathrm{dl}$. During the treatment period (12 weeks), the animals were placed in metabolic cages for 24 h every 4 weeks and urine output and albumin concentration were measured. After 12 weeks of treatment, the animals were weighed, anesthetized with sodium pentobarbitone $(40 \mathrm{mg} / \mathrm{kg}$ i.p.) and blood collected (via cardiac puncture) for measurement of plasma interleukin 6 (IL-6) and serum creatinine levels. The kidneys were weighed and then either snap frozen in liquid nitrogen for protein analysis or immersion fixed with Histochoice (Amresco, Solon, Ohio, USA) for immunohistochemical analysis. The experiments were performed according to the guidelines recommended by the National Institutes of Health and approved by the Georgetown University Animal Care and Use Committee.

Urine Albumin Excretion and Serum Creatinine

Urine albumin and serum creatinine concentrations were determined using the Nephrat II albumin and creatinine companion kits, respectively (Exocell Inc., Philadelphia Pa., USA), according to the manufacturer's protocol. The rate of urine albumin excretion (UAE) was calculated from the measurement of urine albumin concentration and output.

\section{Morphology}

Following fixation, the kidneys were routinely processed to paraffin, sectioned at $4 \mu \mathrm{m}$ and stained with periodic acid-Schiff (PAS, for demonstration of glycogen deposition) or Masson's trichrome (for demonstration of collagen deposition). Glomerulosclerosis was defined as mesangial expansion [15] and the glomerulosclerotic index was assessed in PAS-stained sections using a semiquantitative method as previously described [10]. Tubulointerstitial fibrosis was defined as tubular atrophy or dilatation, deposition of ECM proteins and the presence of inflammatory cells [15]. Tubulointerstitial fibrosis index was assessed in Masson's trichrome-stained sections using a semiquantitative method as previously described [10].

\section{Immunohistochemistry}

Sections were cut at $4 \mu \mathrm{m}$, incubated with $0.1 \%$ albumin and then with antisera against transforming growth factor beta (TGF$\beta, 1: 100$; rabbit polyclonal; R\&D Systems, Minneapolis, Minn., USA), collagen type I (1:100; mouse monoclonal; Sigma), collagen type IV (1:100; goat polyclonal; Southern Biotech, Birmingham, Ala., USA) and CD68 (1:400; mouse monoclonal; Serotec, Oxford, UK) at $4{ }^{\circ} \mathrm{C}$ overnight. The positive immunoreaction was detected using the Envision Plus peroxidase kit (Dako Corporation, Carpentaria, Calif., USA) and by counterstaining with Mayer's hematoxylin. Sections incubated with $0.1 \%$ albumin instead of the primary antiserum were used as negative controls. 
Table 1. Effects of RAL on metabolic and renal parameters

\begin{tabular}{lccc}
\hline & ND & D & D + RAL \\
\hline Body weight, g & $321.4 \pm 28.3$ & $309.2 \pm 35.4$ & $320.0 \pm 17$ \\
Kidney weight, g & $2.7 \pm 0.1$ & $2.7 \pm 0.2$ & $2.9 \pm 0.1$ \\
Blood glucose, mg/dl & $101.1 \pm 9.4$ & $354.9 \pm 41.3^{\mathrm{b}}$ & $342.6 \pm 38.4^{\mathrm{b}}$ \\
Food intake, g/day & $18.7 \pm 3.8$ & $38.4 \pm 5.3^{\mathrm{a}}$ & $32.9 \pm 6.4^{\mathrm{a}}$ \\
UAE, mg/day & $4.2 \pm 0.4$ & $41.3 \pm 9.0^{\mathrm{b}}$ & $21.0 \pm 5.0^{\mathrm{a}, \mathrm{c}}$ \\
Serum creatinine, mg/dl & $0.52 \pm 0.04$ & $0.62 \pm 0.03$ & $0.56 \pm 0.04$ \\
GSI, AU & $0.26 \pm 0.04$ & $1.86 \pm 0.8^{\mathrm{b}}$ & $0.4 \pm 0.06^{\mathrm{d}}$ \\
TIFI, AU & $0.37 \pm 0.05$ & $2.12 \pm 0.5^{\mathrm{b}}$ & $0.20 \pm 0.04^{\mathrm{d}}$ \\
\hline
\end{tabular}

Data are expressed as means \pm SEM; $n=6$ /group. GSI = Glomerulosclerosis index; TIFI = tubulointerstitial fibrotic index; $\mathrm{AU}=$ arbitrary units.

${ }^{\mathrm{a}} \mathrm{p}<0.05,{ }^{\mathrm{b}} \mathrm{p}<0.01$ versus ND.

${ }^{\mathrm{c}} \mathrm{p}<0.05,{ }^{\mathrm{d}} \mathrm{p}<0.01$ versus $\mathrm{D}$.

\section{Western Blotting}

Homogenized samples were loaded on $18 \%$ (TGF- $\beta$ ) or $4-20 \%$ gradient (collagen type I and IV) SDS-PAGE gels and the proteins transferred to a nitrocellulose membrane. The membranes were incubated first with $5 \%$ nonfat milk and then with antisera against TGF- $\beta(1: 1,000)$, collagen type I $(1: 1,000)$ and collagen type IV (1:1,000; mouse polyclonal; Chemicon, Temecula, Calif., USA) at $4^{\circ} \mathrm{C}$ overnight. The membranes were washed, incubated with IgG conjugated to horseradish peroxidase and proteins visualized by enhanced chemiluminescence (KPL, Gaithersburg, Md., USA). The densities of specific bands were normalized to the total amount of protein loaded in each well following densitometric analysis of gels stained with Coomassie blue. The densities of specific bands were quantitated by densitometry using the Scion Image beta (version 4.02) software.

Cytokine Levels

Plasma IL- 6 levels were measured by enzyme-linked immunosorbent assay according to manufacturers' specifications (Biosource International, Camarillo, Calif., USA).

\section{Statistical Analysis}

Data are expressed as means \pm SEM and were analyzed using a one-way ANOVA followed by a Bonferroni posttest, using the Sigma Stat software. Differences were considered significant at $\mathrm{p}<0.05$.

\section{Results}

\section{Body and Kidney Weight, Food Intake and Blood Glucose}

Body and kidney weights were similar in all treatment groups (table 1). The D and D + RAL groups had a 2 -fold increase in food intake compared to ND (table 1). Food intake was similar in the $\mathrm{D}$ and $\mathrm{D}+\mathrm{RAL}$ groups. The $\mathrm{D}$ and D + RAL had a 3.5-fold increase in blood glucose levels compared to the ND animals (table 1).

\section{UAE and Serum Creatinine}

The D rats exhibited a 9.8-fold increase in UAE compared with ND animals (table 1). Treatment with RAL partially attenuated this increase in UAE associated with diabetes; UAE in the D + RAL group was increased 5.0fold compared to ND (table 1). No differences in serum creatinine levels were observed between the treatment groups (table 1).

\section{Morphology}

Kidneys of D animals exhibited mild glomerulosclerosis (fig. 1a) and tubulointerstitial fibrosis (fig. 1b). These kidneys were characterized by deposition of ECM proteins in the glomerulus and tubulointerstitial regions, mesangial expansion, tubular atrophy and infiltration of inflammatory cells (these cells were identified as CD68positive cells, indicating the presence of macrophages (fig. 5a)). The semiquantitative analysis of the degree of glomerulosclerosis and tubulointerstitial fibrosis showed a 7.2-fold increase in glomerulosclerosis and a 5.7-fold increase in tubulointerstitial fibrosis compared with ND (table 1). Treatment with RAL completely attenuated glomerulosclerosis and tubulointerstitial fibrosis compared with ND (fig. 1a, b; table 1).

\section{Collagen Expression}

In the ND kidney, collagen type I was immunolocalized to tubulointerstitial spaces (fig. 2a), while collagen type IV was immunolocalized to basement membranes of proximal and distal tubules and the mesangial areas in the glomerulus (fig. 2b). D was associated with an increase in the intensity of immunostaining for both collagen type I and IV, while treatment with RAL attenuated this increase. Quantitative analysis of collagen type I 
Nondiabetic
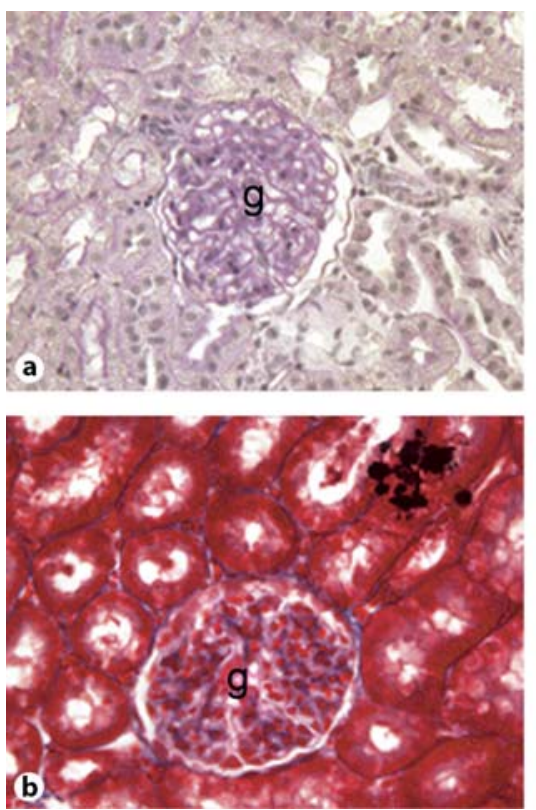

Nondiabetic

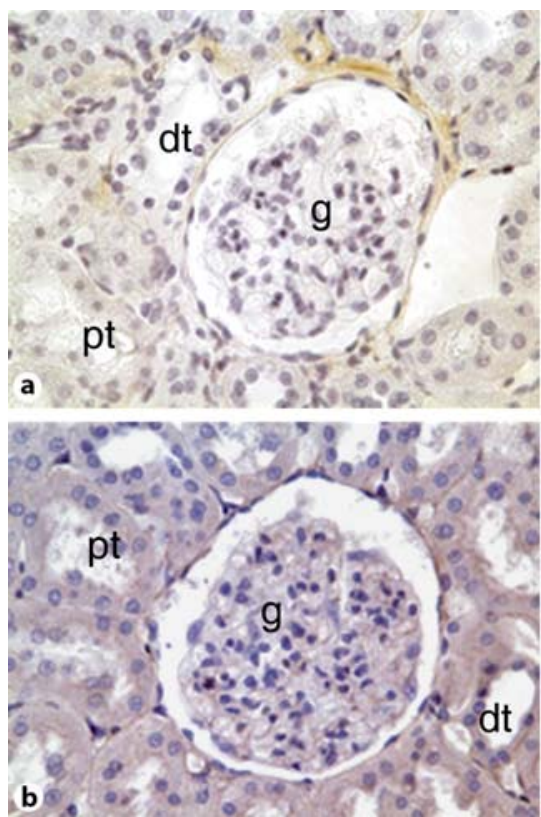

Diabetic
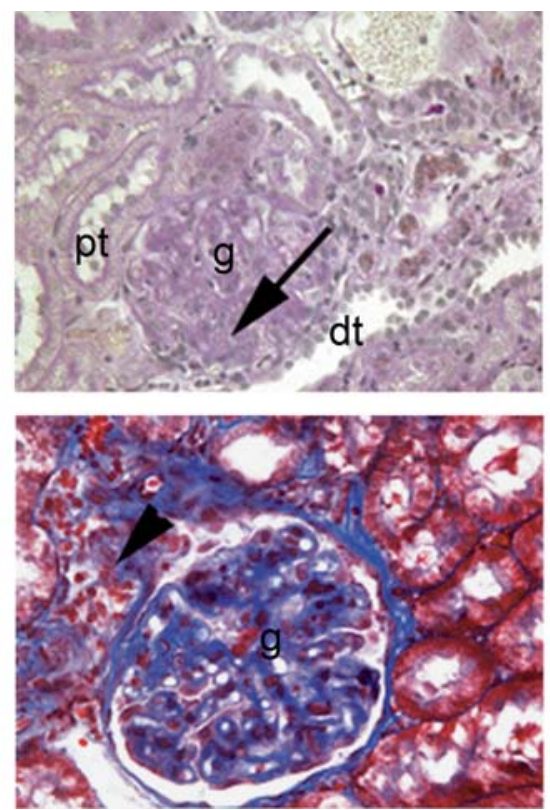

Diabetic
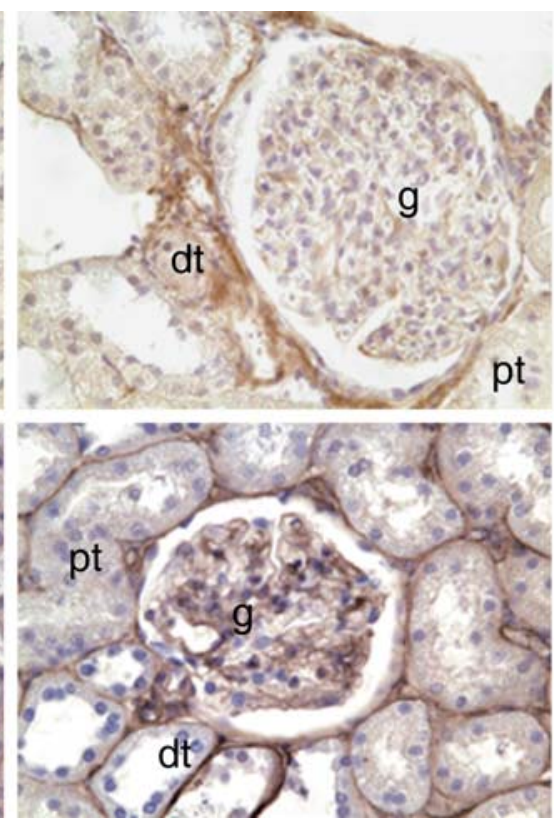

Diabetic + RAL
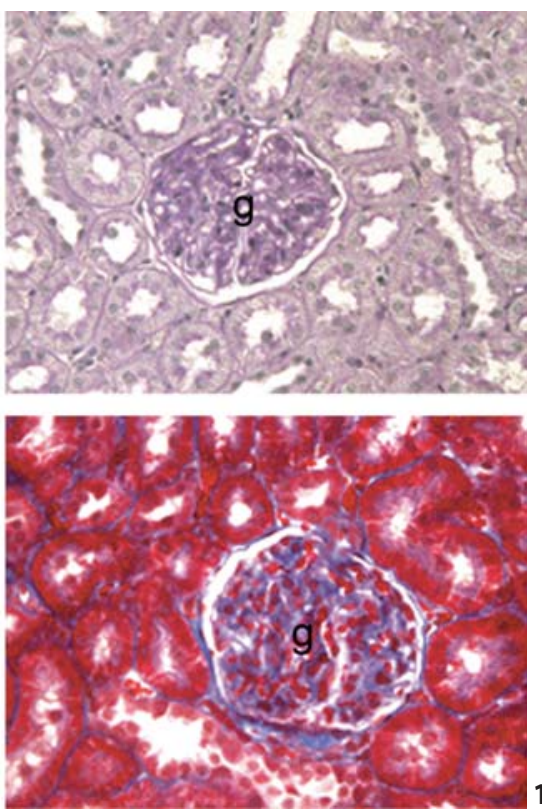

Diabetic + RAL
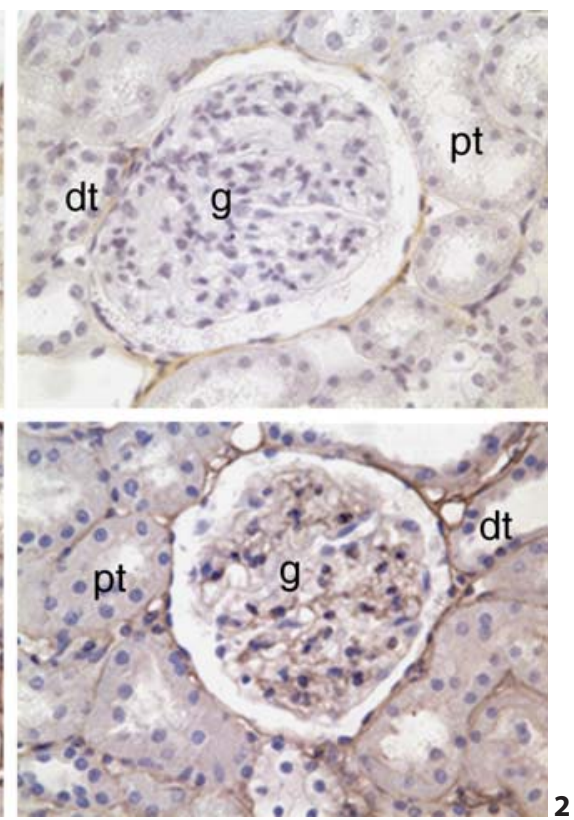

Fig. 1. Renal morphology in ND, D and D + RAL rats. a PASstained sections of the renal cortex. The D kidneys showed moderate glomerulosclerosis (g) characterized by mesangial expansion (arrow). These changes were attenuated by treatment with RAL. b Masson's trichrome-stained sections of the renal cortex. The D kidneys showed moderate tubulointerstitial fibrosis characterized by the presence of ECM deposits (blue staining) and inflammatory infiltrates (arrowhead). These changes were attenuated by treatment with RAL. Original magnification $\times 400$. For abbreviations see legend to Fig. 2.
Fig. 2. Collagen type I and IV immunolocalization in ND, D and $\mathrm{D}+\mathrm{RAL}$ rats. a In the ND kidney, collagen type I (brown staining) was observed in the tubulointerstitium, surrounding the proximal (pt) and distal (dt) tubules. The intensity of immunostaining was increased in the D kidneys, while treatment with RAL decreased the intensity of immunostaining. b In the ND kidney, collagen type IV (brown staining) was observed in the basement membranes in the glomerulus (g), proximal and distal tubules. The intensity of immunostaining was increased in the $\mathrm{D}$ kidneys, while treatment with RAL decreased the intensity of immunostaining. Original magnification $\times 400$. 


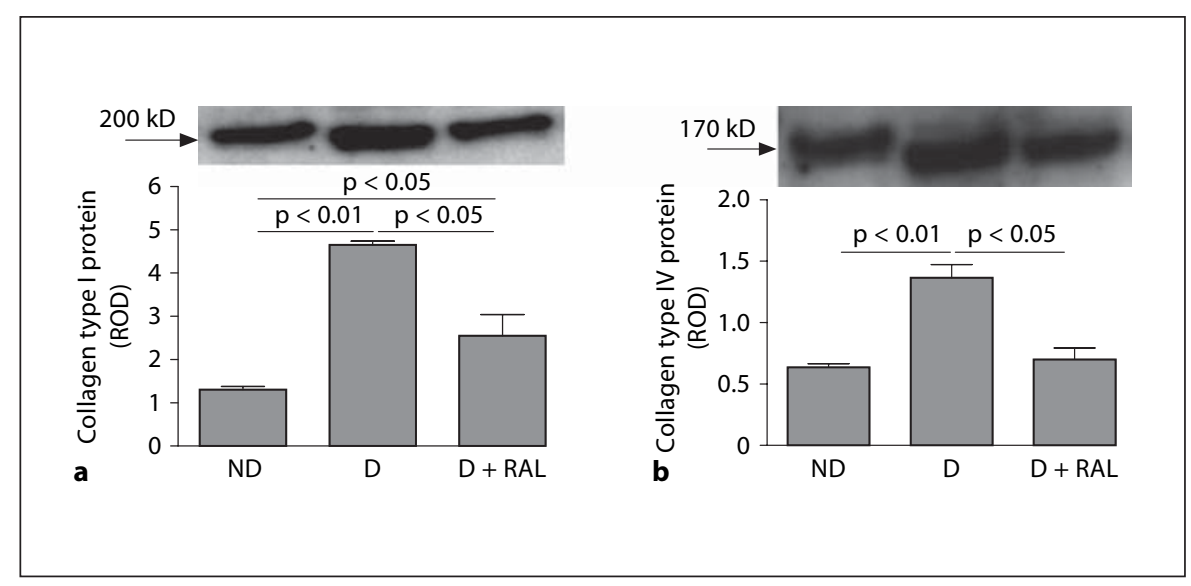

Fig. 3. Collagen type I and IV protein expression in ND, D and D + RAL rats. a In the renal cortex of the D kidneys, collagen type I protein expression was increased 3.6-fold compared to ND. This increase was attenuated by treatment with RAL. b In the renal cortex of the D kidneys, collagen type IV protein expression was increased 2.1-fold compared to ND. This increase was attenuated by treatment with RAL. Top panel in both $\mathbf{a}$ and $\mathbf{b}$ : representative gels of collagen type I and IV protein expression, respectively. Bottom panel in both $\mathbf{a}$ and $\mathbf{b}$ : densitometric scans in relative optical density (ROD) of collagen type I (200-kD band) and IV protein expression (170-kD band), respectively, from top panel. Data are expressed as means \pm SEM. (fig. 3a) and IV expression (fig. 3b) by Western blotting confirmed the immunohistochemistry findings. In the renal cortex of D animals, collagen type I and IV were increased 3.6-fold and 2.1-fold, respectively, while treatment with RAL partially attenuated this increase (fig. $3 a, b)$.

\section{TGF- $\beta$ Expression}

While TGF- $\beta$ was not detectable in the ND kidneys by immunohistochemistry (fig. 4a), it was immunolocalized predominantly in the glomerular mesangial areas in the D kidneys (fig. 4a). Treatment with RAL partially attenuated this diabetes-associated increase in the intensity of TGF- $\beta$ immunolocalization. Western analysis of renal cortical expression of TGF- $\beta$ protein showed a 1.9 -fold increase in the $\mathrm{D}$ animals, while treatment with RAL partially attenuated this increase (fig. $4 \mathrm{~b}$ ).

\section{Renal and Systemic Inflammation}

The D kidney was characterized by the presence of CD68-positive cells, suggesting the presence of macrophages in the in the glomerulus and cortical tubulointerstitium (fig. 5a). Quantitative analysis showed that $\mathrm{D}$ was associated with a 21-fold increase in the abundance of CD68-positive cells compared with ND and that treatment with RAL almost completely attenuated this increase in abundance of CD68-positive cells associated with diabetes (fig. 5b). The $\mathrm{D}$ rats exhibited a 3.5 -fold in- crease in plasma IL-6 levels compared with ND animals (fig. 5c). Treatment with RAL partially attenuated this increase in plasma IL-6 levels associated with diabetes; plasma IL-6 levels were increased by 2.1-fold compared to ND (fig. 5c).

\section{Discussion}

Our previous studies have shown that supplementation with $\mathrm{E}_{2}$ from the onset of diabetes attenuates the development of albuminuria, renal glomerulosclerosis and tubulointerstitial fibrosis [10]. Subsequent studies have shown that the mechanism by which $\mathrm{E}_{2}$ attenuates glomerulosclerosis and tubulointerstitial fibrosis is via increasing ECM degradation and decreasing ECM synthesis [16]. While these studies clearly demonstrate a renoprotective role for $\mathrm{E}_{2}$ in the setting of diabetes, supplementation with $\mathrm{E}_{2}$ longer term has been shown to induce carcinogenesis of uterus and breast $[11,17]$. Furthermore, if supplemented in males, $\mathrm{E}_{2}$ may result in unwanted feminizing side effects. Thus, the aim of the current study was to examine an alternative way of eliciting the renoprotective effects of $E_{2}$ that would eliminate its feminizing and organ side effects. RAL is a SERM that selectively activates estrogen receptors and induces estrogenreceptor-mediated cellular events without the side effects of $E_{2}[12]$. As such, it is an excellent candidate for treat- 

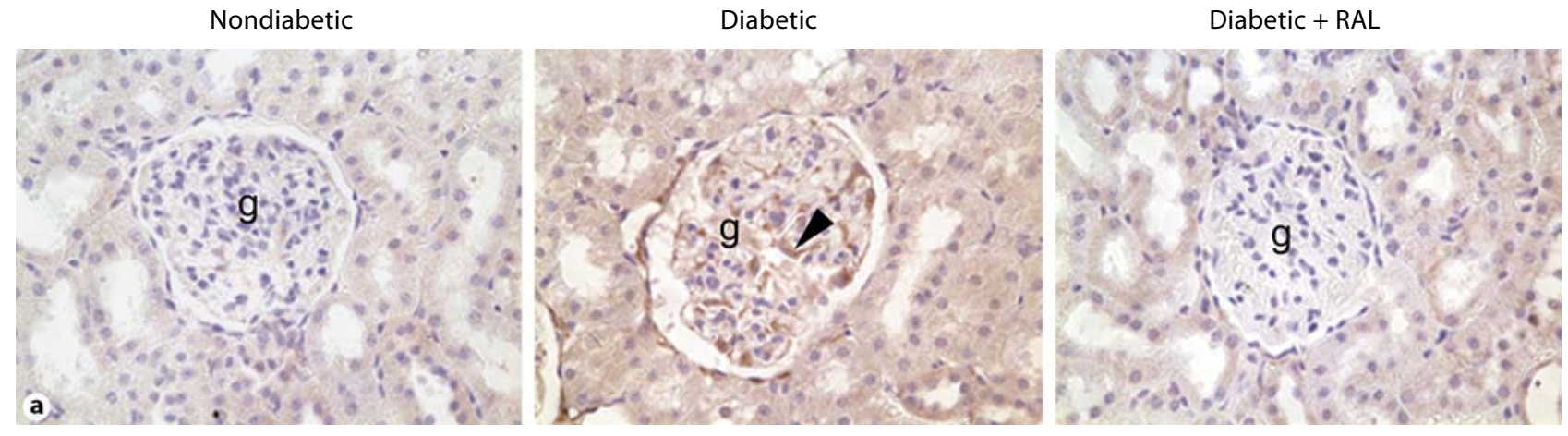

Fig. 4. TGF- $\beta$ renal cortical immunolocalization and protein expression in ND, D and D + RAL rats. $\mathbf{a}$ In the ND kidney, TGF- $\beta$ was not detected by immunohistochemistry. In the D kidney, TGF- $\beta$ (brown staining) was observed predominantly in the mesangial cells (arrowhead) of the glomerulus (g). Similar to ND, TGF- $\beta$ was not detected in the D + RAL group. Original magnification $\times 400$. $\mathbf{b}$ In the renal cortex of the D kidneys, TGF- $\beta$ protein expression was increased 1.9-fold compared to ND. This increase was attenuated by treatment with RAL. Top panel: representative gels of TGF- $\beta$ protein expression. Bottom panel: densitometric scans in relative optical density (ROD) of TGF- $\beta$ protein $(25-\mathrm{kD}$ band) from top panel. Data are expressed as means \pm SEM.
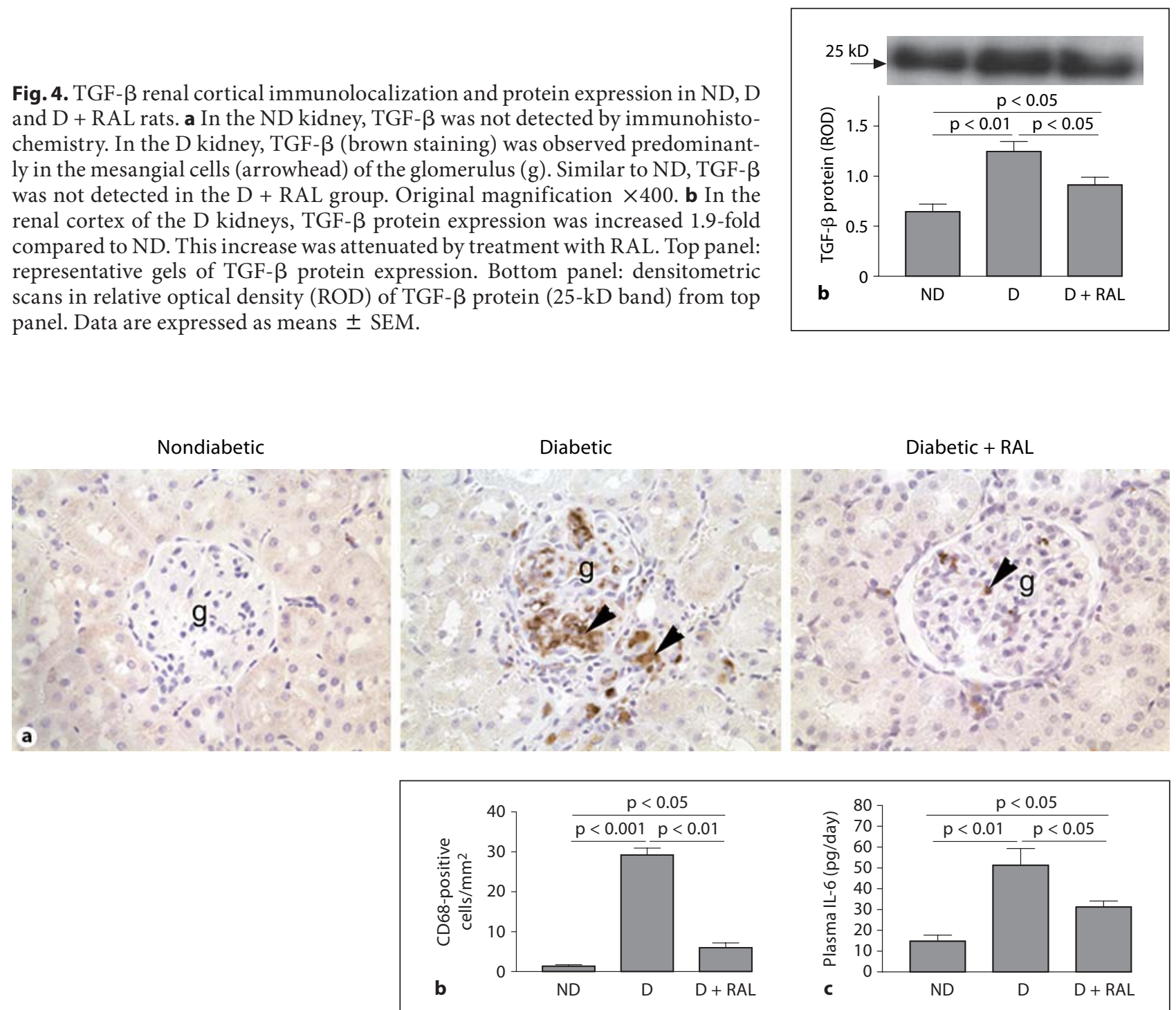

Fig. 5. CD68 immunolocalization and plasma IL-6 levels in ND, D and D + RAL rats. a In the ND kidney, very few CD68positive cells were observed. In the D kidney, CD68-positive cells were evident in the glomerulus (g) and the tubulointerstitium (arrowheads). The abundance of CD68-positive cells was reduced by treatment with RAL. Original magnification $\times 400$. b In the D animals, the abundance of CD68-positive cells was increased 21-fold compared to ND. This increase was attenuated by treatment with RAL. c In the D animals, plasma IL-6 levels were increased 3.5-fold compared to ND. This increase was attenuated by treatment with RAL. Data are expressed as means \pm SEM. 
ment of longer-term organ complications, such as diabetic nephropathy.

Our current study shows that similar to $\mathrm{E}_{2}$, treatment with RAL from the onset of diabetes attenuates diabetesassociated albuminuria, glomerulosclerosis and tubulointerstitial fibrosis. We have found that one of the mechanisms by which RAL attenuates glomerulosclerosis and tubulointerstitial fibrosis is through decreasing collagen type I and type IV protein expression, the two major components of the ECM that are upregulated in diabetic nephropathy. A recent study also found that treatment with RAL reduces expression of another ECM protein, fibronectin, in a model of type II diabetes, namely the $d b / d b$ mouse [14]. In the Cohen diabetic rat, treatment with tamoxifen, an analogue of RAL, reduces diabetes-induced proteinuria and glomerulosclerosis [18]. In cultured renal mesangial cells, tamoxifen reduces collagen type I and type IV synthesis [13]. One of the factors that contribute to increased ECM expression in diabetic nephropathy is TGF- $\beta$ [19]. Our study shows that treatment with RAL decreases diabetes-associated increases in renal cortical expression of TGF- $\beta$. Similarly, the study examining the effects of RAL in the $d b / d b$ mouse has also shown that treatment with RAL reduces TGF- $\beta$ expression in the diabetic kidney. These findings suggest that SERMs are effective in reducing ECM synthesis via reducing TGF- $\beta$ expression and thus may be beneficial in attenuating progressive renal disease without the adverse effects of $E_{2}$ on other organs.

Recent studies have suggested that diabetes is associated with low-grade inflammation and that the diabetic kidney is characterized by the presence of inflammatory cells $[20,21]$. Our studies show that diabetes is associated with increased circulating levels of IL- 6 and infiltration of macrophages in the renal cortex. Treatment with RAL partially attenuated both IL- 6 levels and macrophage infiltration, suggesting that RAL may have anti-inflammatory effects. Recent studies report that treatment with RAL inhibits the expression of chemoattractant molecules and other markers of inflammation in postmenopausal women $[22,23]$. While no studies thus far have examined the anti-inflammatory effects of RAL in the kidney, several suggest that via suppressing the expression of markers of inflammation in the vasculature, including TGF- $\beta$, RAL decreases macrophage recruitment to target sites and thus reduces vascular damage $[24,25]$. Since our study shows that RAL attenuates TGF- $\beta$ expression in the renal cortex, this may be one of the mechanisms by which RAL reduces macrophage infiltration in the diabetic kidney. However, not all studies support the anti-inflammatory role for RAL. In the mouse thymus, in contrast to $E_{2}$, RAL does not modulate $\mathrm{T}$ cell responsiveness and inflammation [26]. Therefore, even though most clinical and experimental studies show an anti-inflammatory effect of RAL, further studies are needed to determine the precise mechanisms through which RAL modulates the complex pathway of immune response and inflammation.

Thus far, very few studies have examined the effects of RAL in diabetes and its associated organ complications. A post hoc analysis of data from the multiple outcomes of RAL evaluation trial, on the effects of 3 years of RAL treatment on glycemic control and cardiovascular risk factors in women with and without type 2 diabetes, has shown that even though RAL does not affect glycemic control, it has favorable effects on total cholesterol, lowdensity lipoproteins and fibrinogen levels $[27,28]$. Studies from this and other laboratories [14] suggest that SERMs are beneficial in attenuating diabetic nephropathy, but whether SERMs have similar protective effects in diabetic cardiomyopathy, neuropathy and retinopathy, remains unknown. The effects of RAL do appear to be organ-specific. Recent findings from the RAL use for the heart trial suggest that treatment with RAL increases the risk of venous thromboembolisms and fatal stroke and decreases the risk of invasive breast cancer and vertebral fracture, while it appears to have no effect on coronary events in postmenopausal women with established or with increased risk for coronary heart disease [29]. These findings stress the importance of considering pre-existing disease and weigh potential benefits against risks when recommending the use of RAL.

While SERMs, including RAL, were originally classified as antiestrogens [30], through activating both estrogen receptor alpha $(\mathrm{ER} \alpha)$ and estrogen receptor beta $(E R \beta)$, SERMs may act as both anti- and proestrogens, depending on the relative abundance of ER $\alpha / E R \beta$ in the target tissue or cell type [12]. In vitro, activation of ER $\alpha$ by $\mathrm{E}_{2}$ has been shown to suppress renal mesangial cell proliferation [31,32] and increase collagen type I degradation $[33,34]$. In contrast, in vivo studies from this and other laboratories suggest that activation of ER $\alpha$ in the diabetic kidney may contribute to glomerular damage [ 9 , 35]. These conflicting observations suggest that ER $\alpha$ may mediate different effects in different renal cells. RAL has a 14-fold higher affinity for ER $\alpha$ than ER $\beta$ [36], suggesting that its effects are mainly mediated through activation of ER $\alpha$. However, it is conceivable that the beneficial effects of RAL observed in the current study are partially due to activation of the ER $\beta$. While ER $\beta$ is only expressed 
in low levels in the diabetic kidney [9], it may be that its largely unexplored actions are critical in regulating renal cell function directly, or by modulating the actions of $\mathrm{ER} \alpha$.

\section{Conclusions}

(1) The present study demonstrates that treatment with RAL from the onset of diabetes is renoprotective by attenuating albuminuria and structural changes associated with diabetes in an experimental model. (2) Further studies are needed to determine if in addition to being able to attenuate albuminuria and structure changes associated with diabetes, treatment with RAL can also reverse these processes once they have already developed, which would render it an effective treatment of diabetic renal complications in females and potentially males.

\section{Acknowledgments}

This work was supported by the Joseph R. Kranin Memorial Young Investigator Research Award from the National Kidney Foundation (Capital area) to C. Maric. The authors would like to thank Eli Lilly for the kind donation of RAL.

\section{References}

1 De Zeeuw D, Remuzzi G, Parving HH, et al: Proteinuria, a target for renoprotection in patients with type 2 diabetic nephropathy: lessons from RENAAL. Kidney Int 2004;65: 2309-2320.

-2 Parving H-H, Tarnow L, Rossing P: Genetics of diabetic nephropathy. J Am Soc Nephrol 1996;7:2509-2517.

-3 Brenner BM, Cooper ME, de Zeeuw D, et al: The losartan renal protection study - rationale, study design and baseline characteristics of RENAAL (Reduction of Endpoints in NIDDM with the Angiotensin II Antagonist Losartan). J Renin Angiotensin Aldosterone Syst 2000;1:328-335.

4 Resnick HE, Howard BV: Diabetes and cardiovascular disease. Annu Rev Med 2002;53: 245-267.

5 Barrett-Connor E, Giardina EG, Gitt AK, et al: Women and heart disease: the role of diabetes and hyperglycemia. Arch Intern Med 2004;164:934-942.

6 Lim SC, Caballero AE, Arora S, et al: The effect of hormonal replacement therapy on the vascular reactivity and endothelial function of healthy individuals and individuals with type 2 diabetes. J Clin Endocrinol Metab 1999;84:4159-4164.

7 Silbiger SR, Neugarten J: The role of gender in the progression of renal disease. Adv Ren Replace Ther 2003;10:3-14.

$>8$ Neugarten J: Gender and the progression of renal disease. J Am Soc Nephrol 2002;13: 2807-2809.

$\checkmark 9$ Wells CC, Riazi S, Mankhey RW, et al: Diabetic nephropathy is associated with decreased circulating estradiol levels and imbalance in the expression of renal estrogen receptors. Gend Med 2005;2:227-237.
10 Mankhey RW, Bhatti F, Maric C: 17beta-Estradiol replacement improves renal function and pathology associated with diabetic nephropathy. Am J Physiol Renal Physiol 2005; 288:F399-F405.

11 Eliassen A, Missmer S, Tworoger S, et al: Endogenous steroid hormone concentrations and risk of breast cancer among premenopausal women. J Natl Cancer Inst 2006;98: 1406-1415.

12 Weatherman R, Clegg N, Scanlan T: Differential SERM activation of the estrogen receptors (ERalpha and ERbeta) at AP-1 sites. Chem Biol 2001;8:427-436.

13 Neugarten J, Acharya A, Lei J, et al: Selective estrogen receptor modulators suppress mesangial cell collagen synthesis. Am J Physiol Renal Physiol 2000;279:F309-F318.

14 Chin M, Isono M, Isshiki K, et al: Estrogen and raloxifene, a selective estrogen receptor modulator, ameliorate renal damage in $d b /$ $d b$ mice. Am J Pathol 2005;166:1629-1636.

15 Animal Models of Diabetic Complications Consortium: Validation of mouse models of diabetic nephropathy. http://www.amdcc. org/.

16 Mankhey R, Wells CC, Bhatti F, et al: 17-beta estradiol supplementation reduces tubulointerstitial fibrosis by increasing MMP activity in the diabetic kidney. Am J Physiol Regul Integr Comp Physiol 2006;292:R769-R777.

17 Rebbeck T, Troxel A, Wang Y, et al: Estrogen sulfation genes, hormone replacement therapy, and endometrial cancer risk. J Natl Cancer Inst 2006;98:1311-1320.

18 Cohen AM, Rosenmann E: Effect of the estrogen antagonist, tamoxifen, on development of glomerulosclerosis in the Cohen diabetic rat. Diabetes 1985;34:634-638.

19 McGowan T, Zhu Y, Sharma K: Transforming growth factor-beta: a clinical target for the treatment of diabetic nephropathy. Curr Diab Rep 2004;4:447-454.
20 Saraheimo M, Teppo AM, Forsblom C, et al: Diabetic nephropathy is associated with lowgrade inflammation in Type 1 diabetic patients. Diabetologia 2003;46:1402-1407.

21 Nguyen D, Ping F, Mu W, et al: Macrophage accumulation in human progressive diabetic nephropathy. Nephrology (Carlton) 2006; 11:226-231.

22 Blum A, Schenke WH, Hathaway L, et al: Effects of estrogen and the selective estrogen receptor modulator raloxifene on markers of inflammation in postmenopausal women. Am J Cardiol 2000;86:892-895.

23 Christodoulakos G, Lambrinoudaki I, Economou E, et al: Circulating chemoattractants RANTES, negatively related to endogenous androgens, and MCP-1 are differentially suppressed by hormone therapy and raloxifene. Atherosclerosis 2006, in press.

24 Seli E, Pehlivan T, Selam B, et al: Estradiol down-regulates MCP-1 expression in human coronary artery endothelial cells. Fertil Steril 2002;77:542-547.

25 Weiss JM, Cuff CA, Berman JW: TGF-beta downmodulates cytokine-induced monocyte chemoattractant protein (MCP)-1 expression in human endothelial cells: a putative role for TGF-beta in the modulation of TNF receptor expression. Endothelium 1999;6:291-302.

26 Erlandsson M, Gomori E, Taube M, et al: Effects of raloxifene, a selective estrogen receptor modulator, on thymus, $\mathrm{T}$ cell reactivity, and inflammation in mice. Cell Immunol 2000;205:103-109.

27 Barrett-Connor E, Ensrud KE, Harper K, et al: Post hoc analysis of data from the Multiple Outcomes of Raloxifene Evaluation (MORE) trial on the effects of three years of raloxifene treatment on glycemic control and cardiovascular disease risk factors in women with and without type 2 diabetes. Clin Ther 2003;25:919-930. 
28 Murase Y, Kobayashi J, Asano A, et al: Effects of raloxifene on adipocytokines in Japanese osteoporotic postmenopausal women with and without type 2 diabetes. Horm Metab Res 2006;38:130-133.

-29 Barrett-Connor E, Mosca L, Collins P, et al: Effects of raloxifene on cardiovascular events and breast cancer in postmenopausal women. N Engl J Med 2006;355:125-137.

-30 Buelke-Sam J, Bryant H, Francis P: The selective estrogen receptor modulator, raloxifene: an overview of nonclinical pharmacology and reproductive and developmental testing. Reprod Toxicol 1998;12:217-221.
31 Neugarten J, Medve I, Lei J, et al: Estradiol suppresses mesangial cell type I collagen synthesis via activation of the MAP kinase cascade. Am J Physiol 1999;277:F875-881.

>32 Dubey RK, Gillespie DG, Keller PJ, et al: Role of methoxyestradiols in the growth inhibitory effects of estradiol on human glomerular mesangial cells. Hypertension 2002;39: $418-424$.

33 Silbiger S, Lei J, Neugarten J: Estradiol suppresses type I collagen synthesis in mesangial cells via activation of activator protein-1. Kidney Int 1999;55:1268-1276.

34 Potier M, Karl M, Zheng F, et al: Estrogenrelated abnormalities in glomerulosclerosisprone mice: reduced mesangial cell estrogen receptor expression and prosclerotic response to estrogens. Am J Pathol 2002;160: 1877-1885.
35 Lovegrove AS, Sun J, Gould KA, et al: Estrogen receptor alpha-mediated events promote sex-specific diabetic glomerular hypertrophy. Am J Physiol Renal Physiol 2004;287: F586-F591.

36 Escande A, Pillon A, Servant N, et al: Evaluation of ligand selectivity using reporter cell lines stably expressing estrogen receptor alpha or beta. Biochem Pharmacol 2006;71: 1459-1469. 